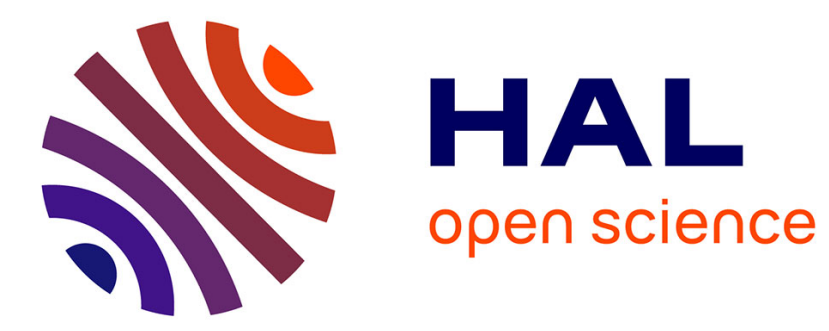

\title{
Three dimensional analysis of a compression test on stone wool
}

\author{
François Hild, Eric Maire, Stéphane Roux, Jean-François Witz
}

\section{To cite this version:}

François Hild, Eric Maire, Stéphane Roux, Jean-François Witz. Three dimensional analysis of a compression test on stone wool. Acta Materialia, 2009, 57, pp.3310-3320. hal-00371354

\section{HAL Id: hal-00371354 \\ https://hal.science/hal-00371354}

Submitted on 27 Mar 2009

HAL is a multi-disciplinary open access archive for the deposit and dissemination of scientific research documents, whether they are published or not. The documents may come from teaching and research institutions in France or abroad, or from public or private research centers.
L'archive ouverte pluridisciplinaire $\mathbf{H A L}$, est destinée au dépôt et à la diffusion de documents scientifiques de niveau recherche, publiés ou non, émanant des établissements d'enseignement et de recherche français ou étrangers, des laboratoires publics ou privés. 


\title{
Three dimensional analysis of a compression test on stone wool
}

\author{
François Hild, ${ }^{a}$ Eric Maire ${ }^{b}$ Stéphane Roux ${ }^{a}$ \\ Jean-François Witz ${ }^{a}$ \\ (a): Laboratoire de Mécanique et Technologie (LMT-Cachan) \\ ENS Cachan / CNRS / UPMC / UniverSud Paris \\ F-94235 Cachan Cedex, France \\ (b): Université de Lyon, INSA-Lyon, MATEIS CNRS UMR 5510 \\ F-69621 Villeurbanne, France.
}

\begin{abstract}
A full three dimensional study of a compression test on a sample made of stone wool is presented. The analysis combines different tools, namely, X-ray microtomography of an in situ experiment, image acquisition and treatment and 3D volume correlation. This set of tools allows for the measurement of three dimensional displacement fields and to evaluate the Poisson's ratio of this type of material. The strain fields are used to analyze localization phenomena. The uncertainty of the analysis is evaluated by actually moving the sample in its undeformed and deformed states, qualifying both image acquisition and correlation steps.
\end{abstract}

Key words: Cellular materials, compression test, deformation heterogeneities, microtomography, 3D image correlation

Email addresses: francois.hild@lmt.ens-cachan.fr (François Hild, ${ }^{a}$ ), eric.maire@insa-lyon.fr (Eric Maire, ${ }^{b}$ ), stephane.roux@lmt.ens-cachan.fr (Stéphane Roux, $\left.{ }^{a}\right)$, jean-francois.witz@lmt.ens-cachan.fr (Jean-François Witz ${ }^{a}$ ). 


\section{Introduction}

$\mathrm{X}$-ray computed microtomography (XCMT) is increasingly used to visualize the complete microstructure of various materials. One of its main advantages lies in the non destructive way of obtaining $3 \mathrm{D}$ views of various materials [1]. By analyzing 3D reconstructed pictures, one has access, for instance, to the structure of biological $[2,3]$ or cellular (either metallic or polymeric foams [4]) materials, and sometimes to the way they deform by using in situ experiments [5] analyzed by 3D correlation algorithms [6-9]. This procedure is to be distinguished from X-ray diffraction which may be used to evaluate elastic strains [10] for crystalline materials. In the present study, the reconstructed scans themselves are used to evaluate full displacement fields.

To measure 3D displacements in the bulk of a scanned material, different routes were followed. First, marker (e.g., W particles) tracking was used to analyze permanent strains in a compression test on aluminum [11]. Second, local correlation techniques were utilized [9, 12, 13], in which small interrogation volumes in two scans are registered. The analysis of the deformation of biomechanical tissues was reported $[7,14]$. Strain fields were also studied in heterogeneous materials $[13,15]$. Third, global (i.e., Galerkin) correlation techniques are an alternative to local approaches. Strain localization in a solid foam was studied with such a technique [9]. When the chosen kinematics is enriched to account for displacement discontinuities, it is possible to measure crack opening displacement fields [16] and profiles of stress intensity factors along the whole crack front [17]. This information is useful, for instance, to model 3D crack propagation.

In the case of entangled materials such as mineral wools, there are very few results on the details of the microstructure and more importantly on the way these materials deform. 2D measurements were performed on the surface of mineral wools $[18,19]$. Heterogeneous deformation modes of high density ma- 
terials occur in uniaxial compression and were mainly controlled by local details of the microstructure such as density [18] and fiber orientation [20]. The latter is crucial when describing the mechanical behavior of high density materials [21]. One of the unanswered questions concerns the representativeness of surface measurements when bulk properties are sought. XCMT provides a unique way of eventually addressing this issue.

The aim of the present paper is to analyze a compression test on a stone wool sample by using XCMT performed with a lab tomograph to analyze the way these materials deform internally. The standardized mechanical performance of those materials refer to a strain level well above the "elastic" limit [22]. In that case, the strain field appears to be generically non uniform, and it is important to characterize the regions that concentrate strains, and possibly identify morphological features that are responsible for such strain concentrations as they appear to be stress (and thus performance) limiting. Full three dimensional strain maps and morphology are therefore extremely valuable. A finite-element based approach to digital image correlation [9] is used to measure displacement and evaluate strain fields during a compression test on stone wool. In Section 2, the studied material, the experimental configuration and the imaging system are presented. The correlation procedure used herein is briefly recalled in Section 3 and its performance is evaluated. The analysis of several images of the same unstrained sample reveals resolution limits resulting from the computed reconstruction. The experimental results are finally analyzed in Section 4 and discussed in Section 5. 


\section{Experimental configuration}

\subsection{Material}

Stone wool is an insulating material made of fibers with basalt-like composition. Figure 1a shows a large scale SEM view of the studied material. The production process of stone wool, called REX, involves (among other mechanisms) a centrifugal instability of a molten rock layer deposited on rapidly spinning wheels. The destabilization of the viscous layer forms a tadpole whose bulky head is drawn by a hot gas burner and whose tail connected to the spinning wheel produces fibers. In the final product, as can be seen in Figure 1a, the fibers are mixed with a fraction of so called "shot" particles (the initial head of the tadpole). These shot particles are a signature of the production process. They increase the density of the final material, and play no role for insulation. The size of the fibers is mostly in the range $1-10 \mu \mathrm{m}$, while the shot particles are of the order of 50 to $100 \mu \mathrm{m}$ in diameter.

Figure 1b shows a typical normalized load vs. macroscopic compressive strain. The latter is evaluated by measuring the relative displacement of the two platens divided by the initial sample height. After an initial non-linear part associated with a gradual contact of the sample surface with both platens, there is an elastic regime followed by another non-linear part, presumably due to frictional slip between fibers. Upon unloading the material in the nonlinear regime, the response is strongly hysteretic as one could expect when solid friction and contact opening occur. 


\subsection{XRCT Setup}

Standard laboratory X-Ray microtomography enables the experimentalist to get $3 \mathrm{D}$ pictures of the local density of solids by exploiting the differential attenuation of a polychromatic X-ray beam while it traverses the analyzed sample [5]. The tomograph used in the present study is made by Phoenix $\mathrm{X}$-ray. It contains two principal components:

- The source is an open transmission nanofocus X-ray tube operated at $90 \mathrm{kV}$ and $140 \mathrm{~mA}$. The thin transmission target bombed by focused electrons is made of tungsten and the focus size is about $6 \mu \mathrm{m}$.

- The X-ray detector is a Paxscan ${ }^{\mathrm{TM}}$ amorphous silicon flat panel initially developed for medical applications. It is composed of about 1900 rows and 1500 lines of sensitive pixels, the size of which is $127 \times 127 \mu \mathrm{m}^{2}$. The pixels are binned to accelerate the acquisition time during the present experiment (final size of the detector: $950 \times 750$ pixels, pixel size: $254 \times 254 \mu \mathrm{m}^{2}$ ).

The source/detector distance is fixed to $80 \mathrm{~cm}$, but the resolution (defined in the present paper as the voxel size in the reconstructed image) can be varied by changing the position of the rotating object between the source and the detector. A good trade-off between sample size and resolution was chosen to be $13.5 \mu \mathrm{m}$ in the present case.

\subsection{Reconstructed volumes}

Image and/or volume correlations require a contrasted texture to measure displacements. The shot particles are extremely helpful for image correlation, as they constitute a random marking with a high contrast evenly distributed within the specimen. As can be seen in Figure 2, they appear as small white clusters with a well defined morphology as compared to the fibers that can- 
not be distinguished individually at the resolution used for this study. The dynamic range of the reconstructed pictures (or scans) is equal to 16 bits. The reconstructed scans are subsequently recoded as 8 bit data. During this recoding, $0.1 \%$ of the black and of the white pixels are intentionally saturated to preserve a good dynamic range, as seen on the histogram of Figure 2. The mean gray level is 43 and the corresponding standard deviation is 27 . The size of the reconstructed volumes is $750 \times 750 \times 750$ voxels, and the analyzed region of interest $(\mathrm{ROI})$ is centered and has a size of $128 \times 128 \times 128$ voxels because of memory limitations (all the results presented herein are performed on a standard PC with a dual core $\mathrm{CPU}$ ). The computation time per couple of analyzed pictures lasted on average ten minutes.

\subsection{In situ compression test}

A testing machine (described in details in Ref. [23]) is clamped on the rotating stage of the tomograph. It allows one to take 3D pictures of the specimen during a compression test. The sample was compressed between two parallel platens with no lubrication. Six scans were acquired for the analyzed test. First, two scans were performed with no applied load. They will be used in the uncertainty analysis (see Section 3.2). Second, four loading steps were considered with a targeted constant displacement between each step. For the last deformation step, the whole experimental setup (sample + compression rig) was moved upwards at constant load and a subsequent acquisition was performed. These two scans will also be used for the uncertainty analysis. The displacement speed was kept rather low $\left(1 \mu \mathrm{m} \mathrm{s}^{-1}\right)$ leading to a strain rate of about $10^{-4} \mathrm{~s}^{-1}$. Due to experimental difficulties, the load could not be measured during this in situ experiment. This will not modify the conclusions of the present paper. 


\section{3D Digital Image Correlation}

In this section, the correlation procedure is first presented. Its performance is evaluated by using two scans of the same sample for which a rigid body motion is applied between the two acquisitions.

\subsection{Finite-element approach to DIC}

Image correlation consists in matching the texture of two pictures (or scans in the present case) with the help of a displacement field to be determined. The passive advection of the texture between the reference $f$ and deformed $g$ images (e.g., see Figure 2) reads from a Lagrangian point of view

$$
f(\mathbf{X})=g(\mathbf{X}+\mathbf{U}(\mathbf{X}))
$$

where $\mathbf{X}$ is the position vector in the reference configuration (i.e., Lagrangian coordinate), and $\mathbf{U}$ the displacement vector. Equation (1) corresponds to the conservation of the optical flow written at a local level, in the reference configuration. In practice, the optical flow conservation hypothesis is not strictly satisfied. This is even more relevant in the case of XCMT for which the 3D scans are the results of a reconstruction procedure that induces some artefacts [1]. Consequently, correlation residuals arise since the difference $f(\mathbf{X})-g(\mathbf{X}+\mathbf{U}(\mathbf{X}))$ does not vanish. From the knowledge of $f$ and $g$, the measurement prob-

lem consists in identifying $\mathbf{U}$ as accurately as possible. To estimate $\mathbf{U}$, the quadratic difference $\varphi^{2}=[f(\mathbf{X})-g(\mathbf{X}+\mathbf{U}(\mathbf{X}))]^{2}$ is integrated over the studied domain in its reference configuration $\Omega$

$$
\Phi^{2}=\int_{\Omega} \varphi^{2} \mathrm{~d} \mathbf{X}
$$

and minimized with respect to the degrees of freedom of the measured displacement field. In the present case, a 3D finite element kinematics is chosen [24] 
for the searched fields $\mathbf{U}$, and the simplest shape functions are used, namely, trilinear polynomials associated with 8-node cube elements (or C8-DIC [9]).

The functional $\Phi^{2}$ is minimized with respect to the set of unknown degrees of freedom. To capture large scale displacements, it is important to perform a first determination of the displacement field based on low-pass filtered images where small scale details are erased. After correction of the deformed picture by this displacement, finer details of the images are restored and the residual displacement computed. This procedure is repeated until the unfiltered images are considered. A very crude filtering is used here because of its simplicity and

efficiency, namely, a filtered image is constructed by gathering each cube of $2 \times 2 \times 2$ voxels into one "super-voxel" and summing their gray level values. A higher degree of filtering is obtained by iterating this procedure. This multiresolution procedure has the additional benefit of decreasing drastically the size of the images to be studied.

\subsection{Uncertainty analyses}

To assess the quality of a correlation result, the correlation residuals are the only data available when the measured displacements are not known. In the following, the normalized correlation residual is considered

$$
\eta(\mathbf{X})=\frac{|\varphi(\mathbf{X})|}{\max _{\Omega}(f)-\min _{\Omega}(f)}
$$

and its mean value $\langle\eta\rangle$ is computed over the whole correlation volume $\Omega$. The uncertainty is conventionally evaluated by the standard deviation of the displacement field $\sigma(U)$. Last, to quantify the strain error, the mean principal strains $\bar{\epsilon}_{i}$ are estimated over the entire region of interest for each rigid body translation. 


\subsubsection{Displacement perpendicular to the rotation axis}

Two scans were experimentally acquired for the same (unloaded) state. Yet, the sample was slightly moved by hand (i.e., $U_{x} \approx 3.1$ voxels, $U_{y} \approx 13.0$ voxels, and $U_{z} \approx 0.3$ voxel, with 1 voxel $\leftrightarrow 13.5 \mu \mathrm{m}$ ) between the two acquisitions to assess the minimum achievable correlation residual and the corresponding performance in terms of strain measurements by correlating the two scans. This procedure allows one to determine the cumulated effect of the correlation algorithm and of the acquisition / reconstruction procedure on the measurement uncertainties. This displacement was mostly performed in the horizontal (XY) plane of the tomograph, perpendicular to the rotation axis i.e., parallel to the platens of the compression rig. The rotation axis $\mathrm{Z}$ during the tomographic scanning is also the compression axis of the in situ tensile rig.

The element size was changed to assess its influence on the overall performance of the correlation algorithm. Figure 3 shows that the larger the element size, the smaller the standard displacement uncertainty. The mean strain levels remain very small and less than $0.05 \%$ in absolute value for all the analyzed cases. The displacement uncertainties level off for element sizes greater than 16 voxels (or $216 \mu \mathrm{m}$ ). In the second regime where a constant level of the displacement uncertainty is observed, it is believed that the saturation is caused by reconstruction artefacts. Figure 4 shows cuts of the residual volume that clearly indicate two ring artefacts when a transverse displacement is performed. The presence of these rings is likely to affect the correlation procedure. This is further confirmed by the fact that the in-plane displacement uncertainties are the only ones that level off.

\subsubsection{Displacement parallel to the rotation axis}

Two scans were also acquired to reproduce the previous analysis for one of the deformed states of the sample. Contrary to the previous case, the translation 
was performed along the loading axis by moving the whole loading setup. Figure 3 shows that the reconstruction procedure clearly impacts differently measurement uncertainties when actual displacements are performed along and perpendicular to the rotation axis. A displacement along the latter leads to a single set of rings in the correlation residuals (Figure 4) and then to a smaller measurement error.

Last, the levels of the correlation residuals with the present analyses constitute minimum levels to be expected when two actual scans are correlated. The fact that they are virtually independent of the element size is all the more important since there will be a single reference level $\langle\eta\rangle=3 \%$.

\section{First analysis of the compression test}

The element size is equal to 16 voxels (or $216 \mu \mathrm{m}$ ) for the first analyses, which is a compromise between the uncertainty level, the spatial resolution (i.e., the element size), and the computation time. Their size is subsequently decreased to enable for a better description of finer displacement details induced by strain heterogeneities. The reference volume is always the same and four loading steps could be analyzed with a reasonable convergence of the algorithm. In Figure 1a, over the SEM view, a box is drawn to show the size of an $\ell=$ 16 voxel element $(216 \mu \mathrm{m})$ in comparison with the microstructure. Each single fiber is not followed; but rather a small bundle of fibers whose kinematics will be assessed.

\subsection{Typical results obtained from the first deformation step}

Prior to the C8 procedure itself, rigid body translations are evaluated by using the cross correlation product calculated via FFTs. The C8 procedure 
is then run recursively at different scales (i.e., by resorting to the coarsegraining procedure discussed above; the latter allows one to capture large displacements and strains $[9,18])$. Figure 5 shows three residual maps. The first one corresponds to the initial picture difference when no corrections are made. There is a clear mismatch between the two analyzed states. When an initial correction estimated as a rigid body translation is performed, it leads to the second map that shows the benefit of this first correction. However, there are still zones in which the residuals are high. Last, the third map shows the residuals at convergence. Except for a few points, the residuals are very low everywhere. This additional test is important, since it is only after having checked that the residual field is small in the whole ROI that the results are definitely deemed trustworthy.

This first deformation step deals with moderate strain levels (ca. -4.5\%) in the elastic regime (Figure 1b). Four different element sizes are considered, namely, $\ell=8$ voxels, 10 voxels, 12 voxels, 16 voxels. The mean correlation residuals (see Table 1) are very close to the value observed in the uncertainty analysis $(\langle\eta\rangle=3 \%)$. The strain levels in the transverse directions remain very small compared with those along the loading direction. In particular, when the element size is less than 16 voxels, the mean transverse strain is less than $0.02 \%$.

\subsection{Second loading step}

The mean strain level is higher in absolute value (ca. -8.1\%) for 16-voxel elements. From this first evaluation, one may want to decrease the element size to capture more accurately local displacement variations. This is made possible by running a further analysis and using the converged solution when the element size is equal to 16 pixels as an initialization. Three elements sizes are considered, namely, $\ell=12,10$, and 8 voxels. No coarse (filtered) image 
and less than 10 additional iterations are needed. Table 1 summarizes the results. As the element size decreases, the mean correlation residual decreases as well. In the present case, it is believed that more degrees of freedom are needed to capture strain heterogeneities. When compared with the reference value (i.e., $\langle\eta\rangle=3 \%$ ), it is concluded that the correlation results with the smallest element size (i.e., $\langle\eta\rangle=3.4 \%$ ) are likely to be closer to the actual displacement field. One possible explanation is that it is the signature of strain heterogeneities requiring a fine mesh to capture more accurately the displacement heterogeneities. The strain heterogeneities might be understood as the consequence of the non-linear regime (Figure 1b) of the material. This point will be further discussed in Section 5 .

\subsection{Third and fourth loading steps}

The third and fourth scans correspond to strain levels for which the nonlinear regime of the material is completely established (Figure 1b). For the third scan, even larger strains occur (ca. -11\%). This level is close to that considered in standardized tests, namely $-10 \%$ [22]. In terms of correlation residuals, there is a small degradation of their levels when compared with those achieved for the first loading step (see Table 1). However, the same trend is observed when the element size decreases, namely, $\langle\eta\rangle$ decreases as well. When compared with the reference value (i.e., $\langle\eta\rangle=3 \%$ ), it is concluded that the correlation results with the smallest element size (i.e., $\langle\eta\rangle=3.8 \%$ ) are still acceptable.

Last, the fourth scan is analyzed. Mean strain levels of the order of $-14 \%$ are reached, and many iterations are needed. The correlation residuals are still acceptable, especially for small element sizes (see Table 1), even though the computation time is significantly larger. This is an indication that at this level, the reference scan should be updated when additional load levels 
are considered, namely, incremental displacement fields between different load levels should be computed from image correlation, and then combined together to compute the total displacement.

\section{Analysis of the whole sequence}

It is of particular importance to note that in the present case no updating was necessary to analyze the four deformation steps and this despite the fact that the mean strain level was rather large (i.e., $-14 \%$ for the last step). This good performance of the correlation procedure is partly due to the fact that only a small ROI is considered, and more importantly, to the multi-resolution procedure. All correlation results converged for mean average displacements between two iterations less than 0.1 mpixel, and mean correlation residuals less than $5 \%$. From the analyzed displacement field, the mean strains are determined over the whole ROI. In the present case, the infinitesimal strain assumption cannot be made. Consequently, a large transformation framework is used. The deformation gradient tensor $\mathbf{F}$ is considered. The latter is related to the displacement $\mathbf{U}$ by

$$
\mathbf{F}=\mathbf{1}+\nabla \mathbf{U}
$$

where $\mathbf{1}$ denotes the second order unit tensor. A polar decomposition of $\mathbf{F}$ is used [25]

$$
\mathbf{F}=\mathbf{R S}
$$

where $\mathbf{R}$ is an orthogonal tensor $\left(\mathbf{R}^{-1}=\mathbf{R}^{t}\right)$ describing the rotations, and $\mathbf{S}$ the right stretch tensor $\left(\mathbf{S}=\mathbf{S}^{t}\right)$. From the latter, the right Cauchy Green tensor $\left(\mathbf{C}=\mathbf{F}^{t} \mathbf{F}=\mathbf{S}^{2}\right)$ allows one to define, for instance, the following strain measures $\mathbf{E}_{m}$

$$
\mathbf{E}_{m}=\frac{1}{2 m}\left(\mathbf{C}^{m}-\mathbf{1}\right)
$$

when $m \neq 0$. In the following, the stretch tensor $\mathbf{S}$ itself is considered, and the corresponding nominal (or Cauchy-Biot) strain $\mathbf{E}_{1 / 2}=\mathbf{S}-\mathbf{1}$ denoted by 
$\boldsymbol{\epsilon}$. When averaged over the whole region of interest, the mean strain is defined as

$$
\bar{\epsilon}=\overline{\mathrm{S}}-1
$$

where $\overline{\mathbf{S}}$ is the main stretch tensor defined via the polar decomposition of the mean deformation gradient $\overline{\mathbf{F}}$

$$
\overline{\mathbf{F}}=\mathbf{1}+\frac{1}{V} \int_{\partial \Omega} \mathbf{U} \otimes \mathbf{N} \mathrm{d} S
$$

and $\mathbf{N}$ denotes the outward normal vector of the external surface $\partial \Omega$ of the ROI $\Omega$, of volume $V$. The principal strains are subsequently determined, and the apparent Poisson's ratio $\nu$ is defined as

$$
\nu=-\frac{\bar{\epsilon}_{2}+\bar{\epsilon}_{3}}{2 \bar{\epsilon}_{1}}
$$

where $\bar{\epsilon}_{1}$ is the contraction strain (i.e., $\left.\left|\bar{\epsilon}_{1}\right|>\left|\bar{\epsilon}_{2}\right|,\left|\bar{\epsilon}_{3}\right|\right)$.

\subsection{Global analysis}

The first step leads to strain levels $\bar{\epsilon}_{1}$ of the order of $-4.2 \%$ when 8 -voxels elements are used. Since the strains $\bar{\epsilon}_{2}$ and $\bar{\epsilon}_{3}$ remain very small (compared with $\bar{\epsilon}_{1}$ ), the evaluation of Poisson's ratio may be difficult. The values of the latter (Table 1) are on average equal to 0.00 with small variations when using large element sizes. This level is to be expected from such type of material for which the entangled and porous texture does not allow for large transverse strain. This result is also observed when a mechanical identification of local elastic parameters is performed on glass wool by using surface measurements only [21] for the same order of magnitude of average strain. The entangled nature of the material explains that it firstly densifies before expanding laterally. Poisson's ratio is reasonably independent of the element size, even if the correlation residuals decrease as the element size decreases (Table 1).

For the second step, the mean strain $\bar{\epsilon}_{1}$ is already large in absolute value 
(ca. $-7.6 \%$ for 8-voxel elements). This level lies above the apparent elastic regime of the material (Figure 1b), yet Poisson's ratio remains very small (0.01). This value is also found for the third step when 8-voxel elements are used. The strain $\bar{\epsilon}_{1}$ increases again in absolute value (ca. $-13.9 \%$ for 8 -voxel elements) when the fourth step is analyzed. Poisson's ratio varies slightly (0.02) when compared with the previous load levels.

Figure 6 shows the longitudinal displacement maps for the whole sequence when a cut of the analyzed ROI is performed by a plane containing the axis of reconstruction. The fours maps are identical in terms of heterogeneity distributions. Only the overall levels vary from step to step. From the first step on, the heterogeneity of the displacement is virtually identical. It is worth noting that the displacement amplitudes are at least two orders of magnitude greater than the measurement uncertainties (Figure 3).

\subsection{Strain heterogeneities}

The previous displacement fields are analyzed in terms of the component $F_{z z}-$ $1=U_{z, z}$. If only small rotations occur, as is likely in the present case at the level of each considered element (Figure 1a), this quantity corresponds to the longitudinal nominal strain $\epsilon_{z z}$ (see Equation (7)). In the following, the values $\epsilon_{z z}^{e}$ are estimated as averages per element of $F_{z z}-1$. This is made possible by the use of Equation (8) applied element-wise. This value is assigned to the middle point of each element and the fields shown hereafter correspond to trilinear interpolations of these "nodal" quantities.

Figure 7 shows the maps of $\epsilon_{z z}^{e}$ for the four steps. Strain heterogeneities are well marked since the beginning of the experiment and the overall distribution remains identical throughout. Figure 8 shows the incremental strain maps $\Delta \epsilon_{z z}^{e}$ for the same sequence. This last information allows one to visualize the 
"active" parts of the deformation process. In the present case, the third and fourth steps lead to more uniform fields compared with the first and second steps, thereby confirming the conclusions drawn from the analysis of the total strain and displacement maps.

Stone wool, akin to many cellular solids, exhibits a strongly non-linear mechanical behavior in compression. As it densifies, solid friction between fibers would lead to a plateau stress, if it was not balanced by the hardening effect due to the multiplicity of inner contacts created in compression (Figure 1b). Consequently, the fiber length between contacts is reduced, and hence the stiffness (directly related to this length) increases rapidly. Therefore, any density heterogeneity present in the unloaded state is expected to lead to significant strain heterogeneity. Conversely, in compression, it is expected that density heterogeneities are to progressively vanish. This is consistent with the previous observations, where incremental strains tend to become more uniform under load (Figure 8), while the total strain seems to saturate to a state of fixed contrast (Figure 7).

It is to be noted that the previous argument is only a "mean-field" view of the problem. In reality, the volume elements are not all subjected to the same stress, and the latter itself is dependent both on the local properties of the element, but also on its surrounding. The strain compatibility is a global constraint that makes the analysis more complex. In particular, Figure 7 shows that large strain elements form a pattern that has to span through the entire sample.

To better understand the spatial distribution of this pattern, a 3D visualisation showing the clustering of large strains in $3 \mathrm{D}$, after thresholding $\epsilon_{z z}$ is shown in Figure 9-a. The high strain regions are more or less oriented with an oblique angle compared to the compression direction, which is vertical in this figure. Figure 9-b shows for the same sample, oriented in the same way as in Figure 9- 
a, the outline of the regions where the density in the initial sample (i.e., before deformation) are low (the threshold is here applied to capture the darkest regions of the tomographic scan). Given the origin of the contrast in these images, low gray levels indicate low densities. The spatial pattern is again complex. The regions do not correspond perfectly but the specific oblique angle is the same in both figures.

Let us stress the difference with the phenomenon of localization in metals and alloys, which also reflects an uneven strain distribution but that usually reveals an instability. For stone wool, the reverse is true, namely, heterogeneity is progressively erased under load and incremental strains tend to be increasingly homogeneous. To investigate this question further, a correlation between density and (axial) strain is searched for. By averaging strains for elements of mean density belonging to successive intervals (Figure 10), a strong decrease of compressive strain with density is observed. As the deformation level increases (in absolute value), the same trend is observed, yet shifted in the log-log plot. The same type of conclusion was drawn when pictures of the surface of light density glass wool were analyzed [18]. Note that the gray levels give a rather direct indication of absorption, and hence density, but absolute densities are scaled and offset to provide a slightly saturated histogram (Figure 2b) at the reconstruction stage.

Unfortunately, it is not possible to be more quantitative without performing a full numerical modeling of the material. Even in a statistical sense, for a heterogeneous elastic material, there is no unique relationship between local strain and local elastic modulus. The microstructure and stiffness spatial correlations affect crucially such a link. However, the fact that lighter volumes are more compressed than denser ones is likely, and supports the observation that incremental strains tend to become increasingly uniform under increasing load. 


\section{Summary}

It was shown that in situ mechanical tests can be performed in a laboratory tomograph, and processed from digital image correlation techniques to evaluate quantitatively global mechanical properties, such as Poisson's ratio. The uncertainty quantification methodology and results are presented to make explicit the choice of the correlation parameters to be used (such as the element size), and to decide when the correlation procedure can be trusted. These procedures are fairly general and applicable to a wide variety of materials. Furthermore, strain heterogeneities could be captured even for large strain levels with a good confidence in the results thanks to the correlation residuals.

For stone wool, Poisson's ratio evaluations at different load levels were performed, and remain consistently equal to 0 (at the scale of the accuracy of the analysis) for compressive strains of the order of $10 \%$. Very low values were found on a global level, in agreement with local elastic analyses [21]. This result confirms the representativeness of surface observations to evaluate the bulk properties. Last, it was shown that the material density was responsible for local heterogeneities in strains, and that a correlation between local (in the sense of the measurement discretization) density and strains could be performed. This result was already obtained with 2D pictures of mineral wool surfaces. It allows us to validate the fact $2 \mathrm{D}$ analyses, which are simpler, give a good account of the bulk properties of mineral samples. Other local features not considered herein, such as the local anisotropy (already investigated in 2D

studies), are presumably important to propose a quantitative identification of the local elastic properties [20] based upon 3D bulk measurements, as those proposed herein. 


\section{Acknowledgments}

This work was part of the project PHOTOFIT funded by "Agence Nationale de la Recherche." The stone wool sample was kindly provided by Jean-Baptiste Rieunier (Saint-Gobain Isover).

\section{References}

[1] Baruchel J, Buffière J-Y, Maire E, Merle P, Peix G. X-Ray Tomography in Material Sciences. Hermes Science, Paris (France), 2000.

[2] Ambrose J, Hounsfield GN. Br. J. Radiol. 1973;46:148

[3] Hounsfield GN. Br. J. Radiol. 1973;46:1016

[4] Bernard D, editor. 1st Conference on 3D-Imaging of Materials and Systems 2008. ICMCB, Bordeaux (France), 2008.

[5] Maire E, Buffière J-Y, Salvo L, Blandin J-J, Ludwig W, Létang J-M. Adv. Eng. Mat. 2001;3:539

[6] Bart-Smith H, Bastawros A-F, Mumm DR, Evans AG, Sypeck DJ, Wadley HNG. Acta Mater. 1998;46:3583

[7] McKinley TO, Bay BK. J. Biomech. 2003;36:155

[8] Viot P, Bernard D, Plougonven E. J. Mater. Sci. 2007;42:7202

[9] Roux S, Hild F, Viot P, Bernard D. Comp. Part A 2008;39:1253

[10] Sinclair R, Preuss M, Maire E, Buffière J-Y, Bowen P, Withers PJ. Acta Mater. 2004;52:1423

[11] Nielsen SF, Poulsen HF, Beckmann F, Thorning C, Wert JA. Acta Mater. $2003 ; 51: 2407$

[12] Bay BK, Smith TS, Fyhrie DP, Saad M. Exp. Mech. 1999;39:217

[13] Bornert M, Chaix J-M, Doumalin P, Dupré J-C, Fournel T, Jeulin D, Maire E, Moreaud M, Moulinec H. Inst. Mes. Métrol. 2004;4:43

[14] Bay BK. J. Orthopaedic Res. 1995;13:258 
[15] Lenoir N, Bornert M, Desrues M, Bésuelle P, Viggiani G. Strain $2007 ; 43: 193$

[16] Réthoré J, Tinnes J-P, Roux S, Buffière J-Y, Hild F. C.R. Mécanique 2008;336:643

[17] Rannou J, Limodin N, Réthoré J, Gravouil A, Ludwig W, BaïettoDubourg M-C, Buffière, Combescure A, Hild F, Roux S. Submitted for publication 2008

[18] Hild F, Raka B, Baudequin M, Roux S, Cantelaube F. Appl. Optics 2002;IP 41:6815

[19] Bergonnier S, Hild F, Roux S. J. Strain Analysis 2005;40:185

[20] Bergonnier S, Hild F, Roux S. J. Mat. Sci. 2005;40:5949-59

[21] Witz J-F, Roux S, Hild F, Rieunier J-B. J. Eng. Mat. Tech. 2008;130:021016-1

[22] Thermal insulating products for building applications - Determination of compression behaviour. European standard EN 826, 1996

[23] Buffière J-Y, Maire E, Cloetens P, Lormand G, Fougères R. Acta Mater. $1999 ; 47: 1613$

[24] Zienkievicz OC, Taylor RL. The Finite Element Method. McGraw-Hill, London (UK), 1989.

[25] Truesdell C, Noll W. The Non-Linear Field Theories of Mechanics. In: Flügge S, editor. Handbuch der Physik, vol. III/3. Springer-Verlag, Berlin, 1965. 


\section{List of Tables}

1 Effect of the element size on the correlation results for the four deformation steps. 
Table 1

Effect of the element size on the correlation results for the four deformation steps.

\begin{tabular}{|c|c|c|c|c|c|}
\hline$\ell$ (voxels) & $\langle\eta\rangle$ & $\bar{\epsilon}_{1}$ & $\bar{\epsilon}_{2}$ & $\bar{\epsilon}_{3}$ & $\nu$ \\
\hline 16 & $3.5 \%$ & $-4.5 \%$ & $0.0 \%$ & $0.1 \%$ & 0.01 \\
\hline 12 & $3.3 \%$ & $-4.3 \%$ & $0.0 \%$ & $0.0 \%$ & 0.00 \\
\hline 10 & $3.2 \%$ & $-4.2 \%$ & $0.0 \%$ & $0.0 \%$ & 0.00 \\
\hline 8 & $3.1 \%$ & $-4.2 \%$ & $0.0 \%$ & $0.0 \%$ & 0.00 \\
\hline \hline 16 & $4.0 \%$ & $-8.1 \%$ & $0.0 \%$ & $0.3 \%$ & 0.02 \\
\hline 8 & $3.4 \%$ & $-7.6 \%$ & $0.0 \%$ & $0.1 \%$ & 0.01 \\
\hline \hline 16 & $4.6 \%$ & $-11.4 \%$ & $0.0 \%$ & $0.6 \%$ & 0.03 \\
\hline 8 & $3.8 \%$ & $-10.7 \%$ & $0.0 \%$ & $0.3 \%$ & 0.01 \\
\hline \hline 16 & $4.9 \%$ & $-14.5 \%$ & $0.1 \%$ & $1.0 \%$ & 0.04 \\
\hline 8 & $3.9 \%$ & $-13.8 \%$ & $0.1 \%$ & $0.5 \%$ & 0.02 \\
\hline
\end{tabular}




\section{List of Figures}

1 (a) Large scale SEM view of the analyzed sample showing the fibers and coarse shot particles. The box drawn on the right hand top corner shows the size of a 16-voxel element (where the voxel size refers to the tomographic image shown in Figure 2a) as a reference. (b) Typical normalized load vs. macroscopic compressive strain of the studied stone wool. The arrows show the mean strain levels at which the DIC analysis was performed.

2 Region of interest of the reference 3D image (a) of compressed stone wool sample $(128 \times 128 \times 128$ voxels, 8 -bit digitization $)$ and corresponding histogram (b).

3 Standard displacement uncertainty $\sigma(U)$ as a function of the element size when transverse and longitudinal displacements are prescribed (1 voxel $\leftrightarrow 13.5 \mu \mathrm{m})$.

4 Cuts about the center of the region of interest along three perpendicular directions of residual maps at convergence $f-g$ (expressed in gray levels) when transverse (left) or longitudinal (right) displacements of the whole setup are performed. 
5 Correlation residuals $|f-g|$ expressed in gray levels, when no correlation is performed (a), when only rigid body translation is accounted for (b), and at convergence (c) after the whole correlation procedure was carried out. This last residual shows that the registration was successful when comparing it to the deformed scan (d) and the reference scan shown in Figure 2. All positions are for the same voxel frame as that used in Figure 2.

6 Longitudinal displacement maps $U_{z}$ expressed in voxels (1 voxel $\leftrightarrow 13.5 \mu \mathrm{m})$ for the first four loading steps when 8-voxel elements are used.

7 Deformation gradient component $\epsilon_{z z}^{e}$ for the first four loading steps.

8 Incremental deformation gradient $\Delta \epsilon_{z z}^{e}$ for the four loading steps.

$93 \mathrm{D}$ isosurface to highlight the regions where the measured compression strain is the highest (a), and where the gray level of the reconstructed images are low, i.e., with the smaller density (b). The strains were obtained by using the sample in its first deformed state, and the gray level for the sample in its initial state (i.e., before deformation).

10 Log-log plot of the average axial compressive strain vs. initial average density (in gray levels) for the first $(\bullet)$, second $(\times)$, third (*) and fourth (o) deformation steps. A dotted line of slope -1 is shown as a guide to the eye. 


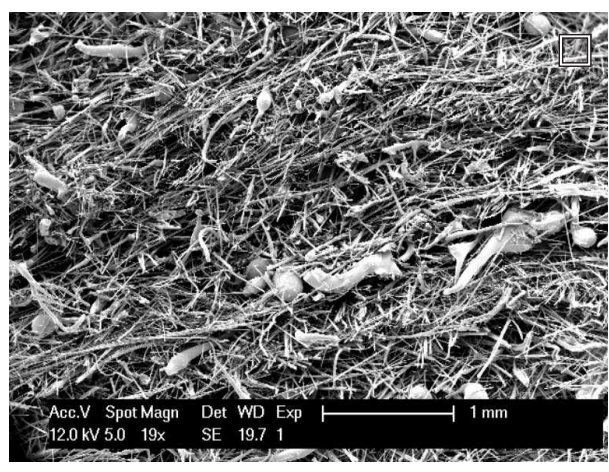

$-a-$

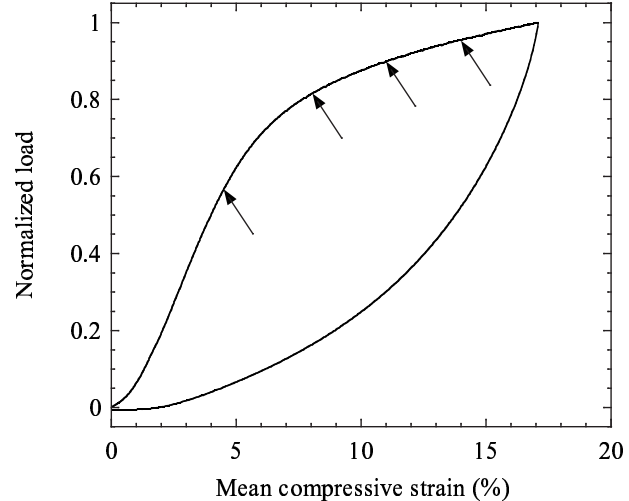

$-b-$

Fig. 1. (a) Large scale SEM view of the analyzed sample showing the fibers and coarse shot particles. The box drawn on the right hand top corner shows the size of a 16-voxel element (where the voxel size refers to the tomographic image shown in Figure 2a) as a reference. (b) Typical normalized load vs. macroscopic compressive strain of the studied stone wool. The arrows show the mean strain levels at which the DIC analysis was performed. 

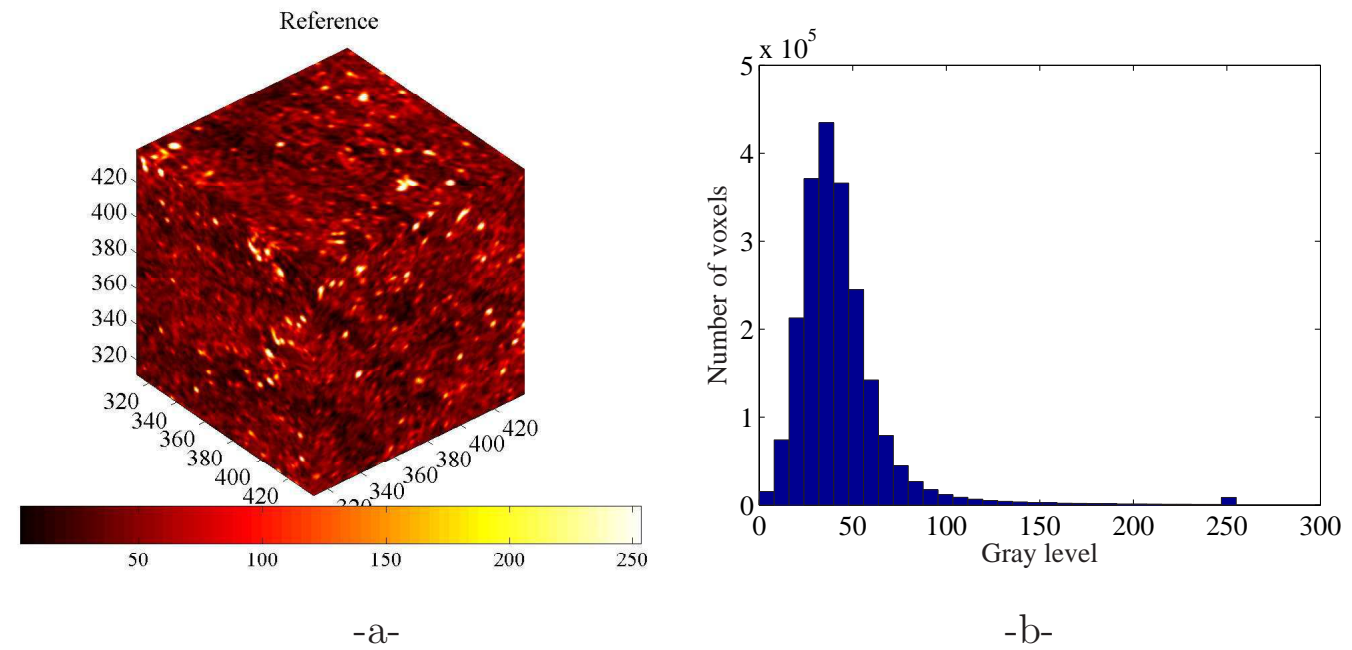

Fig. 2. Region of interest of the reference 3D image (a) of compressed stone wool sample $(128 \times 128 \times 128$ voxels, 8 -bit digitization $)$ and corresponding histogram (b). 


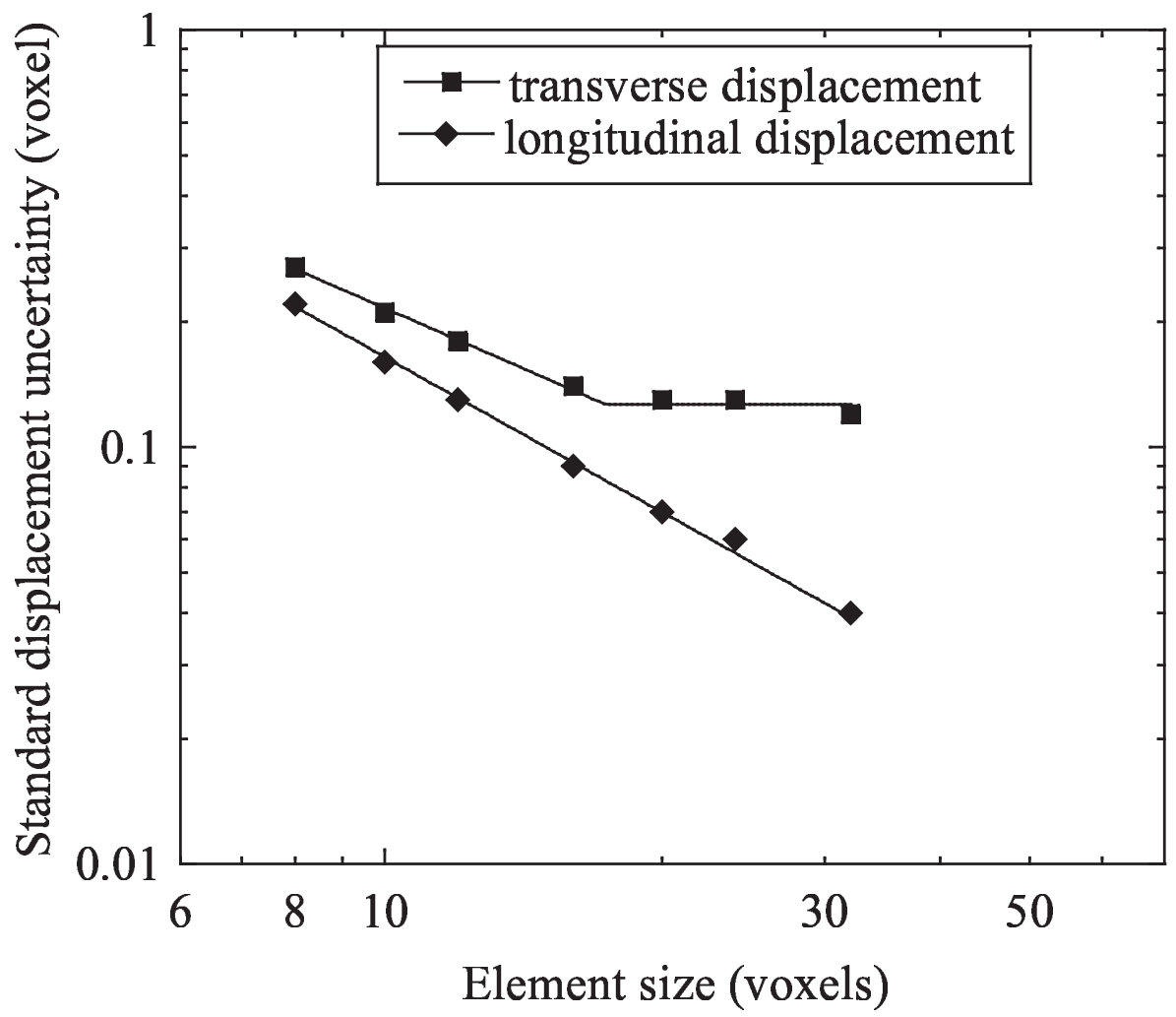

Fig. 3. Standard displacement uncertainty $\sigma(U)$ as a function of the element size when transverse and longitudinal displacements are prescribed ( 1 voxel $\leftrightarrow 13.5 \mu \mathrm{m})$. 

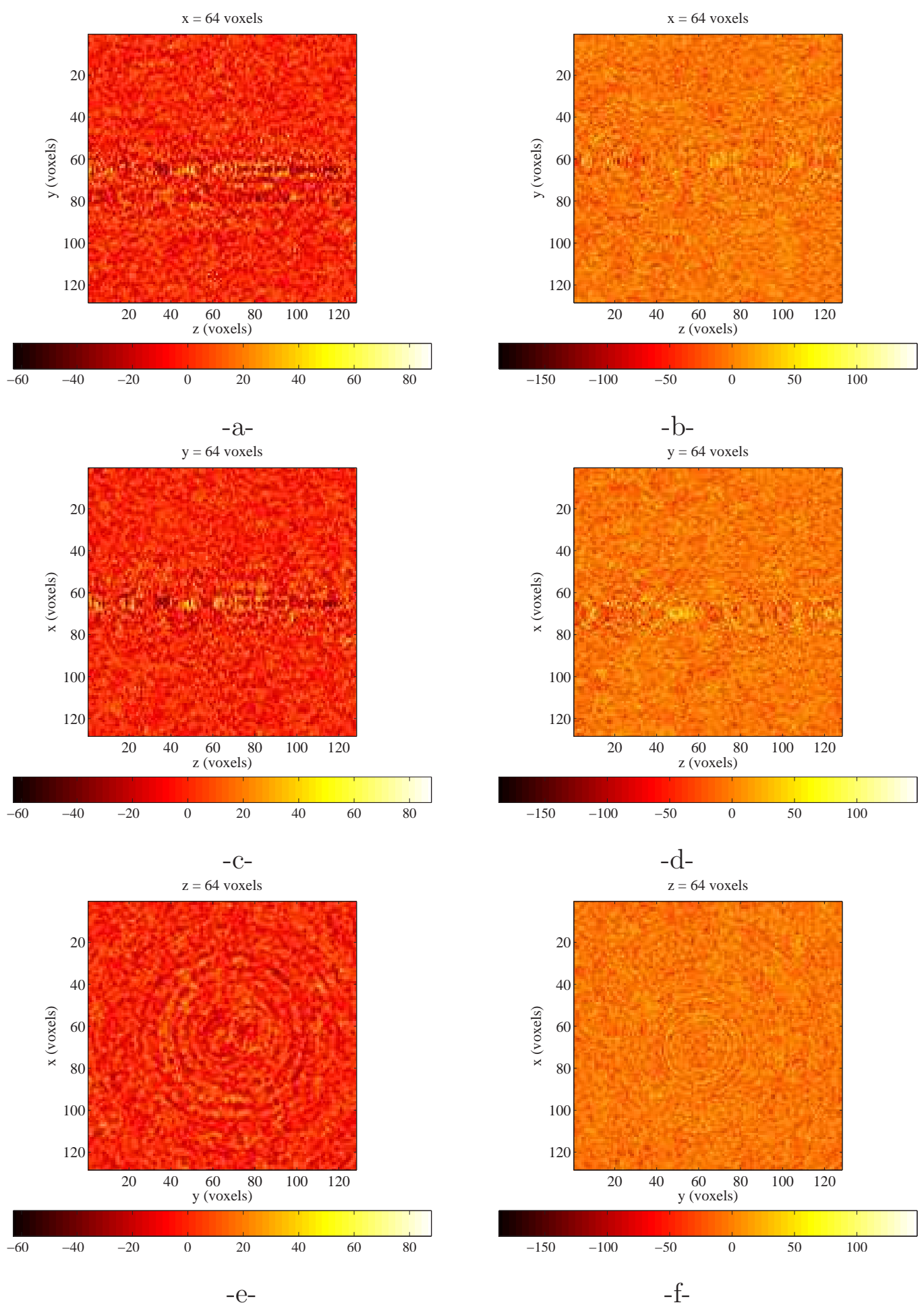

Fig. 4. Cuts about the center of the region of interest along three perpendicular directions of residual maps at convergence $f-g$ (expressed in gray levels) when transverse (left) or longitudinal (right) displacements of the whole setup are performed. 

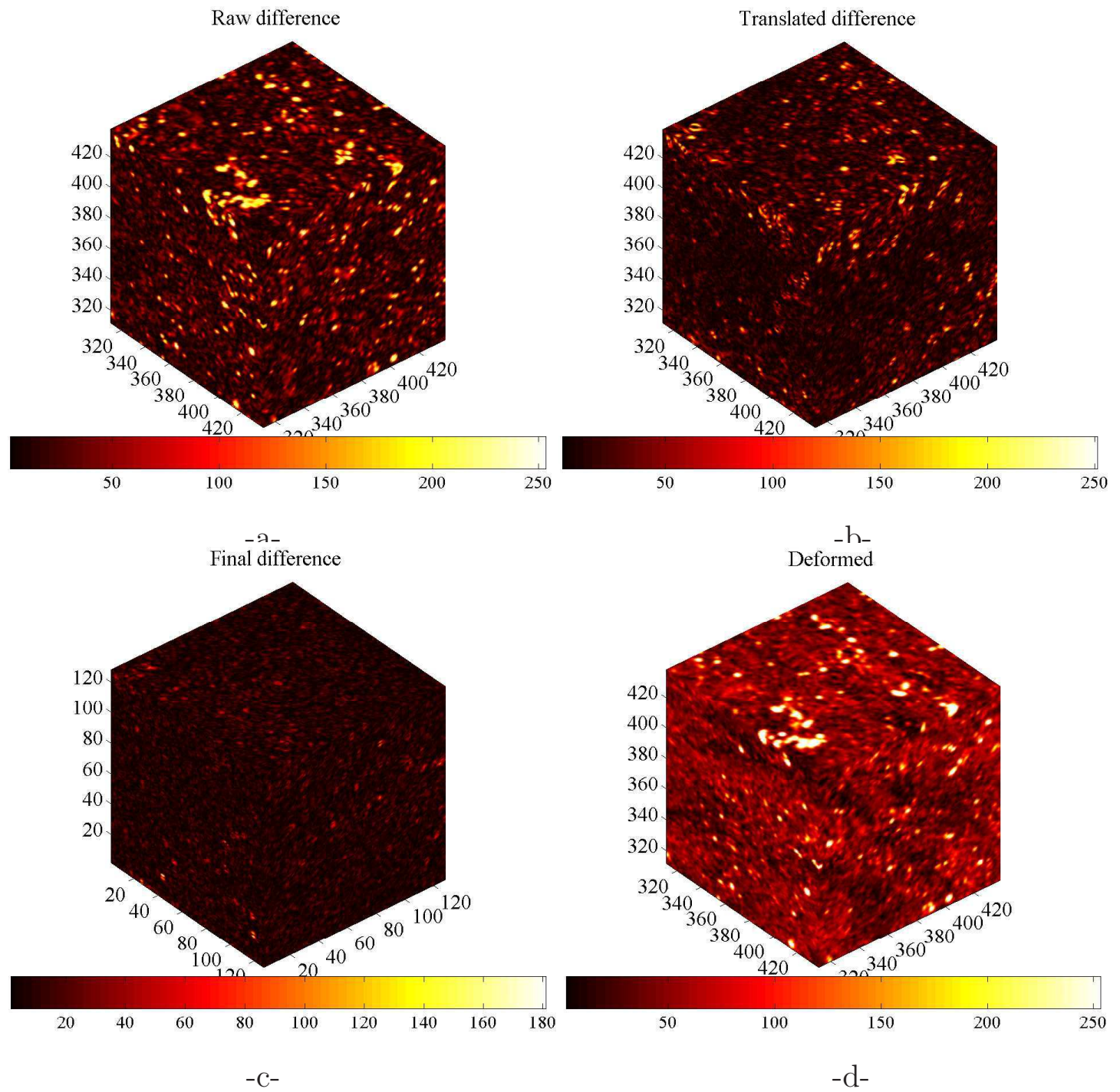

Fig. 5. Correlation residuals $|f-g|$ expressed in gray levels, when no correlation is performed (a), when only rigid body translation is accounted for (b), and at convergence (c) after the whole correlation procedure was carried out. This last residual shows that the registration was successful when comparing it to the deformed scan (d) and the reference scan shown in Figure 2. All positions are for the same voxel frame as that used in Figure 2. 

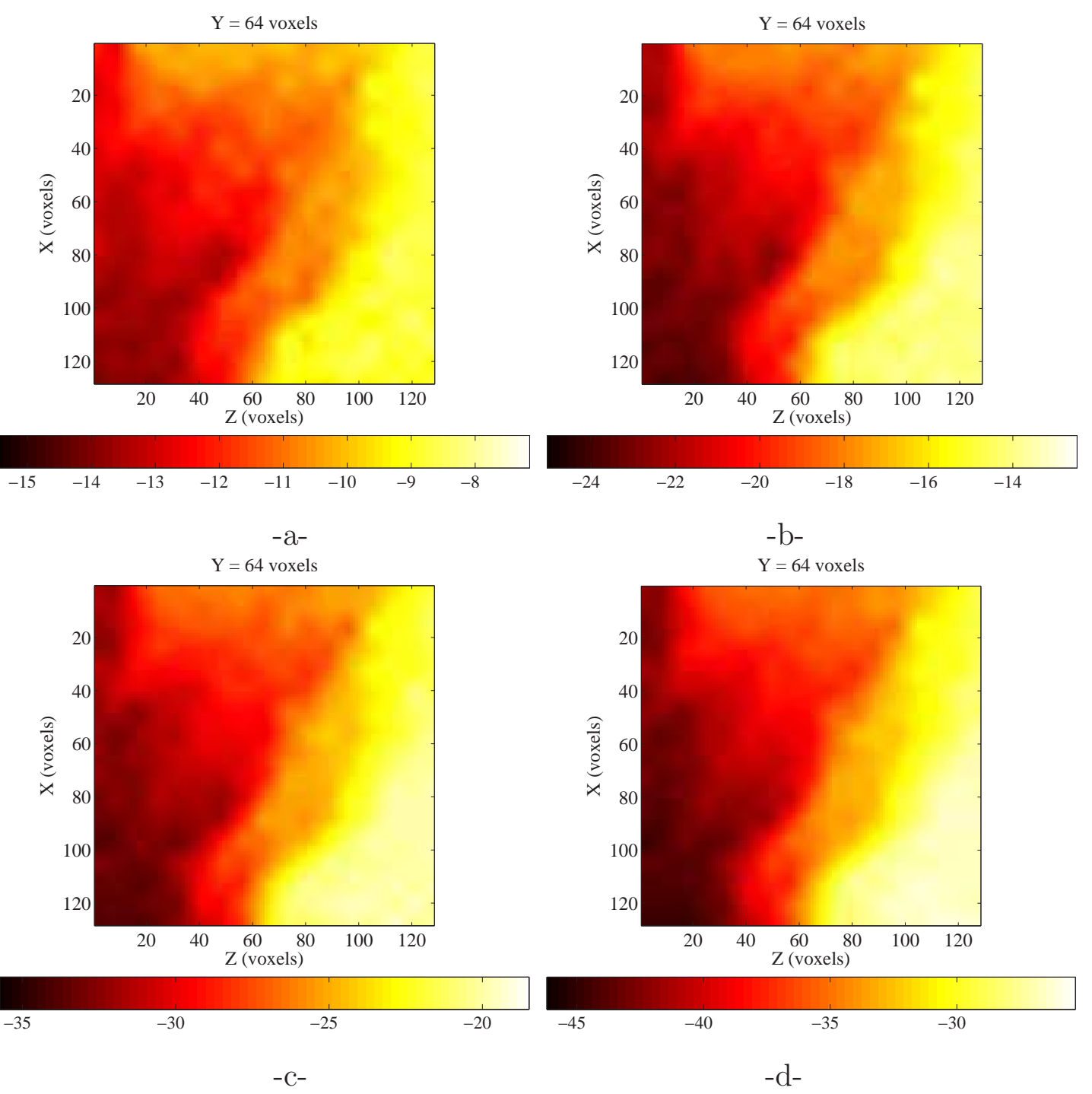

Fig. 6. Longitudinal displacement maps $U_{z}$ expressed in voxels (1 voxel $\left.\leftrightarrow 13.5 \mu \mathrm{m}\right)$ for the first four loading steps when 8-voxel elements are used. 

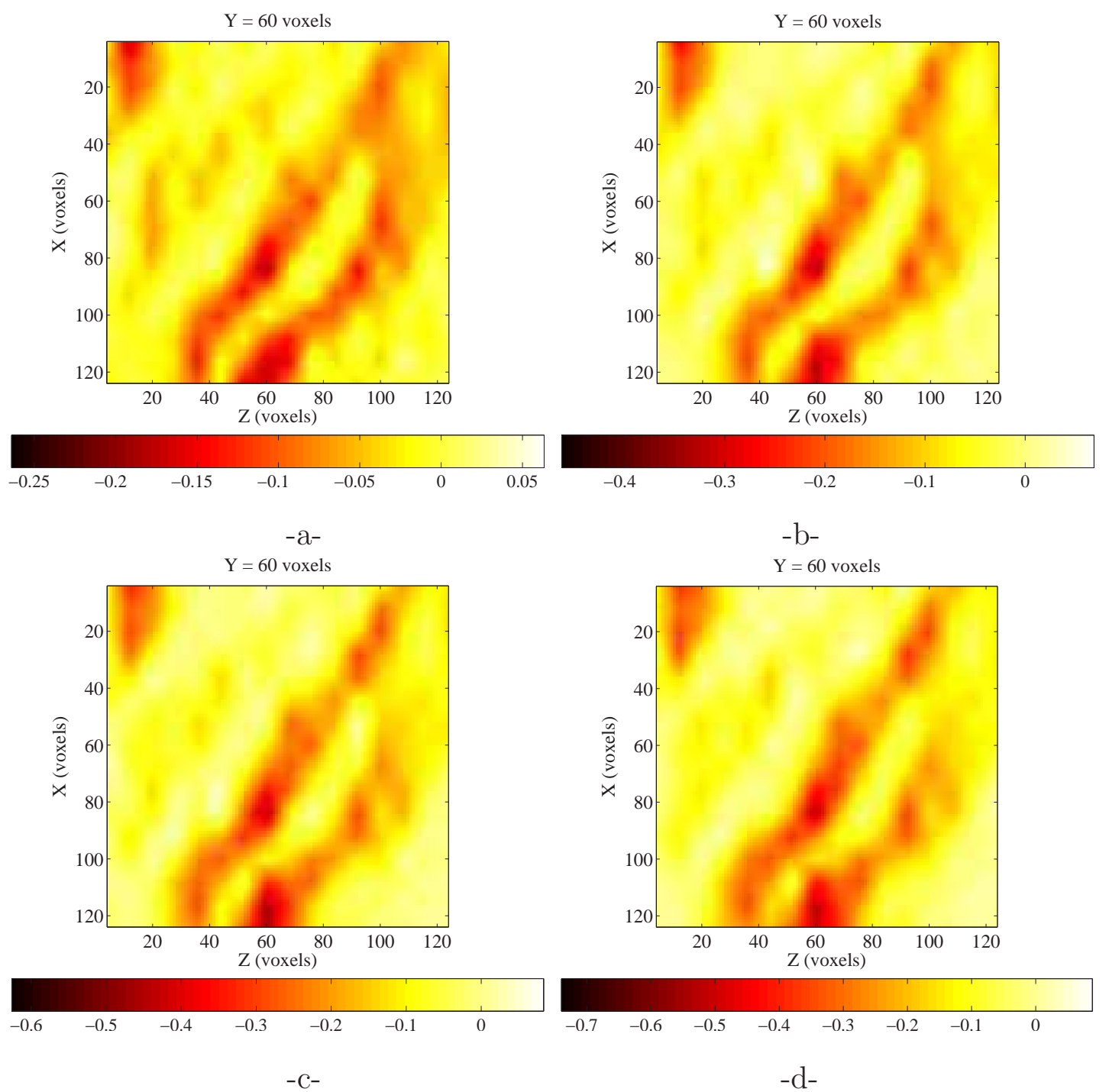

Fig. 7. Deformation gradient component $\epsilon_{z z}^{e}$ for the first four loading steps. 

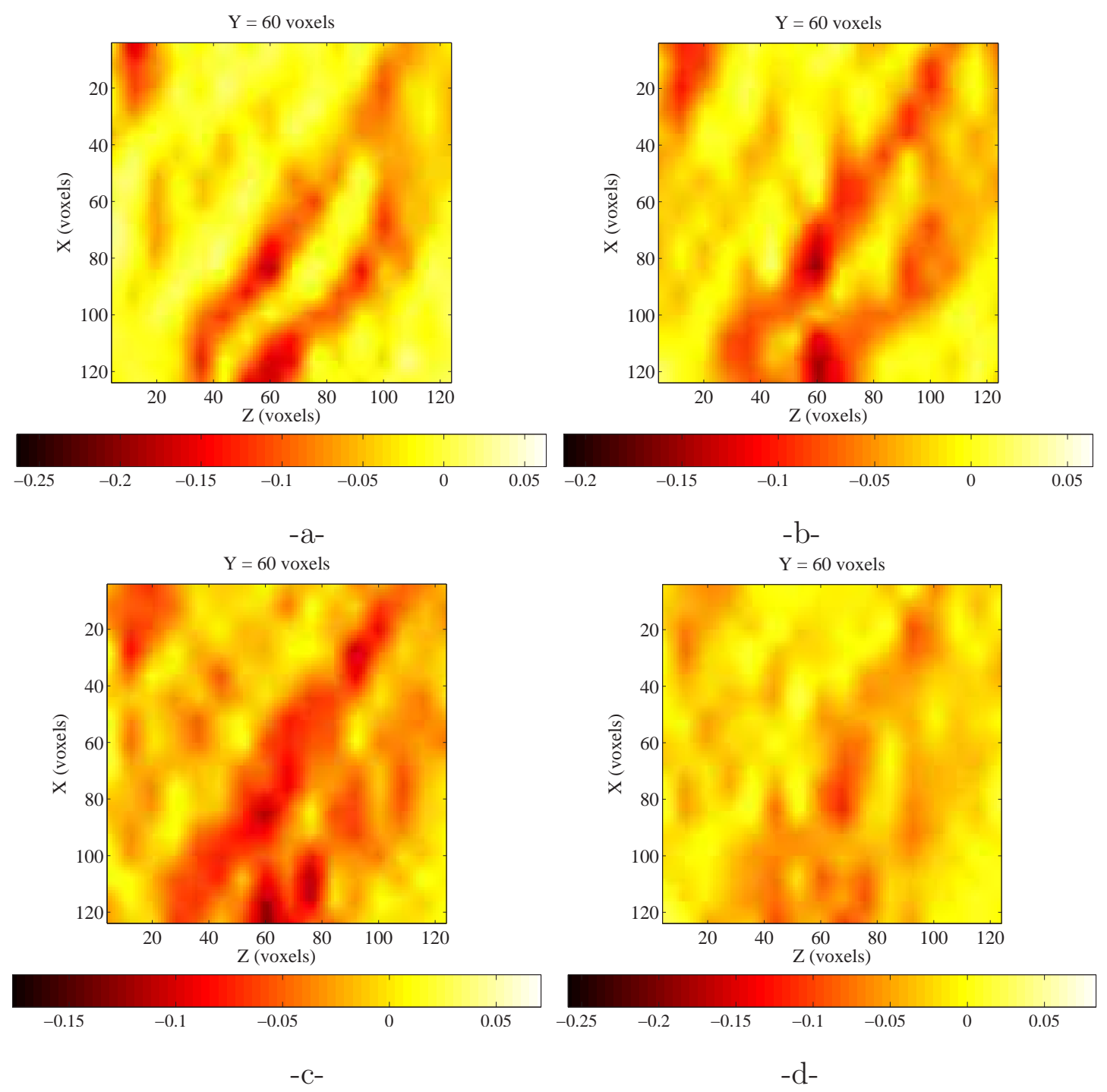

Fig. 8. Incremental deformation gradient $\Delta \epsilon_{z z}^{e}$ for the four loading steps. 


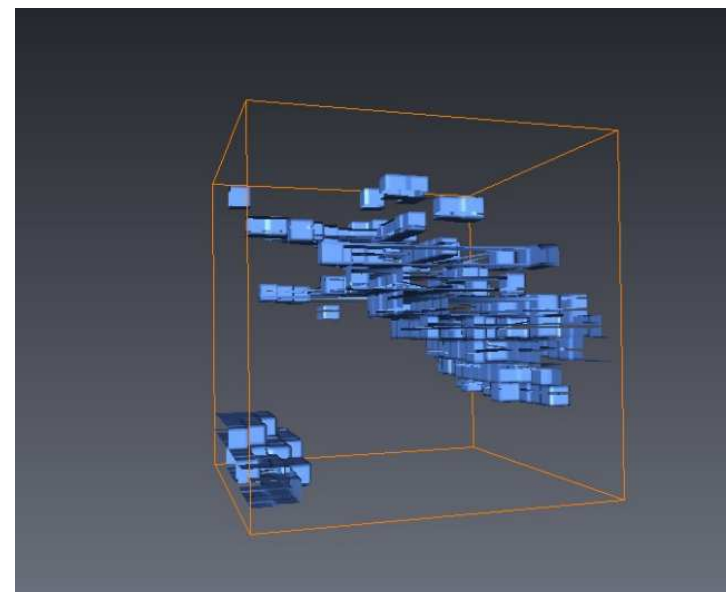

$-a-$

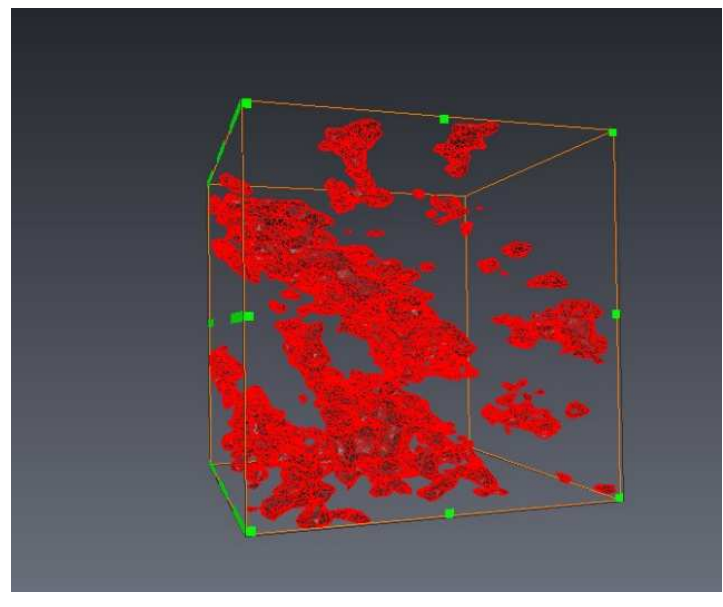

$-\mathrm{b}-$

Fig. 9. 3D isosurface to highlight the regions where the measured compression strain is the highest (a), and where the gray level of the reconstructed images are low, i.e., with the smaller density (b). The strains were obtained by using the sample in its first deformed state, and the gray level for the sample in its initial state (i.e., before deformation). 


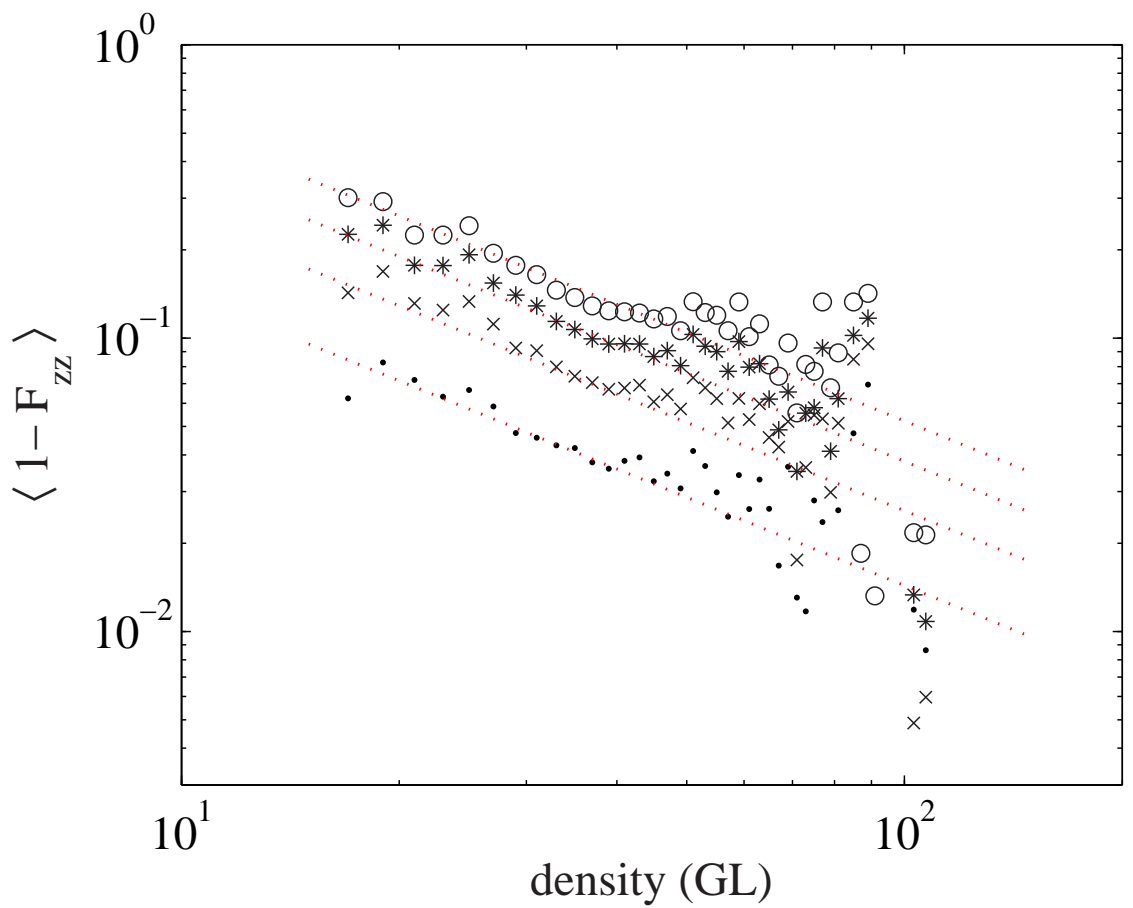

Fig. 10. Log-log plot of the average axial compressive strain vs. initial average density (in gray levels) for the first $(\bullet)$, second $(\times)$, third $(*)$ and fourth $(\circ)$ deformation steps. A dotted line of slope -1 is shown as a guide to the eye. 